\title{
Female Sexual Dysfunction in Married Medical Students
}

\author{
Alaleh Asghari Roodsari*, Afsaneh Khademi, $\mathrm{MD}^{\dagger}$, Ehsan Akbari Hamed, \\ Seideh Leila Tabatabaiifar, Ashraf Alleyassin, $\mathrm{MD}^{\dagger \dagger}$
}

\begin{abstract}
:
Background: Sexuality and its manifestation constitute some of the most complex of human behavior. Sexual dysfunction is more prevalent in women than in men. Prevalence of the subgroups of female sexual disorders is: desire disorder in $\mathbf{5 - 4 6 \%}$, arousal disorders in $\mathbf{7 - 1 0 \%}$ and orgasmic disorders in 7 $10 \%$. The objective of our study was to measure the prevalence of female sexual dysfunction in female medical students. Materials and Methods: Thirty two medical students participated in the study. The mean age was $24.30 \pm 1.29$ years. Duration of marriage was $2.68 \pm 1.5$ years. Their husbands' education ranges from secondary school diploma to $\mathrm{PhD}$. Persian version of Sexual Function Questionnaire (SFQ) was piloted among medical students with and without chief complaint of female sexual dysfunction. Results: Prevalence of an abnormal score in each subgroup of SFQ was as follows: $20.0 \%$ in desire, $56.7 \%$ in arousal sensation, $33.3 \%$ in arousal lubrication, $36.7 \%$ in orgasm, $6.7 \%$ in pain and $20.0 \%$ in enjoyment. In our study $\mathbf{4 0 . 0 \%}$ had sexual problems at least in one subgroup and $6.7 \%$ had problems in all subgroups. Only 2 participants were unsatisfied with their sexual life and seeking for any treatment. Discussion: In this study, prevalence of Female Sexual Dysfunction (FSD) ranges from 6.7\% to $56.7 \%$ in subgroups of the disorder. Solving social problems have critical effect on quality of life. Evaluation of FSD is important in total and especially in women who are university educated and will be occupied in essential positions.
\end{abstract}

KEY WORDS: female sexual dysfunction, medical student, sexual function questionnaire

\section{BACKGROUND}

Sexuality and its manifestations constitute some of the most complex of human behavior. The expression of sexuality and intimacy remains important throughout the life span (1). We know that female genital sexual response is a combination of vaso-congestive and neuromuscular events in the genital tract and pelvic floor which are controlled in part, by specific

* To whom correspondences should be addressed: Alaleh Asghari Roodsari, 8th floor, 1st Entrance, 8th Block, Apadana complex, Tehran, Iran. E.mail: atulip_a@yahoo.com.

$\dagger$ Assistant Professor. Department of Obstetrics and Gynecology/ Shariati Hospital / Tehran University of Medical Sciences

$\dagger \dagger$ Associate Professor. Department of Obstetrics and Gynecology/ Shariati Hospital / Tehran University of Medical Sciences neurotransmitters (2). Female sexual response begins with desire (libido) for sexual interaction. Masters and Johnson (3) in 1966 described four component of the sexual response: excitement, plateau, orgasm and resolution. But the act of sex includes a woman's sexual self and self-image, intimate relationships, family, society and culture. The complexities of her environment, sexual and partner history, past relationships, mental health status, current medical problems and hormonal status all play a role (4).

Sexual dysfunction is defined as "disturbances in sexual desire and the psycho-physiological changes that characterize the sexual response and cause marked distress and interpersonal difficulty"(5). It is a 
combination of problems that has both biologic and psychosocial components and is multi-factorial in etiology. Until recently, the diagnosis of female sexual dysfunction (FSD) has relied on the classification system of the American Psychiatric Association's Diagnosis and Statistical Manual of Mental Disorders, 4th ed. (DSM-IV). This classification was expanded to include psychogenic and organic cause of desire, arousal, orgasm and sexual pain disorders. $(4,6)$ New questionnaire such as Sexual Function Questionnaire (SFQ) was designed to respond to this need.

Hypoactive sexual desire disorder is the persistent or recurrent deficiency of sexual fantasies, thoughts and/or desire for or receptivity to sexual activity which cause personal distress. Female sexual arousal disorders (FSAD) include the persistent or recurrent inability to attain or maintain sufficient sexual excitement, causing personal distress. An orgasmic disorder is the persistent or recurrent difficulty, delay in or inability to attain orgasm following sufficient sexual stimulation and arousal causing personal distress. Sexual pain disorders include dyspareunia, vaginismus and non-coital sexual pain disorders (4).

Most studies suggest that sexual dysfunction is more prevalent in women than in men. A comprehensive literature review by Simons and Carey (7) notes an overall prevalence of the following disorders: desire disorders 5-46\%; arousal disorders 7-10\% and orgasmic disorders $7-10 \%$. A study by Shokrollahoi et al in Iran was done and revealed the prevalence of inhibited desire $15 \%$, inhibited orgasm $26 \%$, lack of lubrication $15 \%$, vaginismus $8 \%$ and dyspareunia $10 \% ; 38 \%$ of the women had at least one sexual dysfunction. The most common sexual difficulties reported were "too little foreplay before intercourse" and" partner chooses inconvenient time" (8\% each) (8).

Several measurements have recently been published and include the Female Sexual Function Index (FSFI) (9), the Female Sexual Questionnaire (SFQ) (6), and the Female Sexual Distress Scale (FSDS) (10).The FSFI was supported by Zonagen, Inc. and Bayer AG. It is 19item questionnaire assessing six domains including desire, subjective arousal, lubrication, orgasm, satisfaction and pain. The SFQ, developed by Pfizer for clinical trials, is a 31 -item survey that measures seven domains of female sexual function (desire, enjoyment, orgasm, arousal-sensation, arousal-lubrication, pain and partner satisfaction) (4). The FSDS was supported by Derogatis, et al. The original version has 20 -item and a "polished" version has 12-item questionnaire assessing frequency or intensity of female sexual distress. It is important to remember that the prevalence data is dependent on the assessment techniques and these have been highly variable (7). SFQ is a multidimensional questionnaire which is utilized in many studies and clinical trials all over the world. SFQ was incorporated in four European, multi-center, phase II clinics and in USA (6).

The objective of our study was to determine prevalence of FSD among married medical students. We chose this group due to omit the effect of age, disease, and level of education as confounding factors.

\section{MATERIALS AND METHODS}

In this pilot study during spring 2004, 32 married medical students at Tehran University of Medical Sciences from different sociocultural levels were enrolled. Women in this study were aged 21-26 years (mean age \pm SD: $24.30 \pm 1.29$, range: $21-29$ ).

We devised a questionnaire including: demographic data: female age, husband's age, husband's education, duration of marriage, past obstetrical history, past medical history, drug history during past 6 months, hirsutism, acne, pattern of menses, contraception method used, body mass index (BMI) and complaints of FSD (by clinical interview) (Table 1). Subsequently, SFQ-V1 questionnaire was completed by each subject.

Subjects with a history of ingesting drugs for more than 3 months in the past 6 months, endocrinologic diseases and psychiatric problems were excluded. Two were excluded because of past history of Diabetes mellitus and antidepressant use.

SFQ-V1 has 6 domains and 26 items. These 6 domains included desire, arousal-sensation, arousallubrication, orgasm, enjoyment and pain (6) (Table 2). For providing Persian version of SFQ -V1, a translation was produced and back-translated to English, to ensure that the original meaning of each item was maintained. Persian version of SFQ was circulated among married medical students. The prevalence of sexual dysfunction was calculated for each domain. The congruence of scores and demographic data were estimated.

This study was approved by the ethics committee of Tehran University of Medical Sciences. Data were evaluated with the statistical software package SPSS (release 10; Chicago, IL). Significant level sets at $\mathrm{P}<0.05$.

\section{RESULTS}

Our subjects were medical students from the first to seventh levels. Husband's education ranges from secondary school diploma to $\mathrm{PhD}$. All of the subjects were nulliparous. Four (13.3\%) of subjects had acne. Hirsutism was found in $3(10 \%)$. Hormonal assays were normal in these patients. In $40.0 \%$ the contraception method utilized was condom, $16.7 \%$ used oral contraceptives and $43.30 \%$ didn't use any contraception (Table 1). 
Only $2(6.6 \%)$ participants were unsatisfied about their sexual life in clinical interview and sought treatment.

Table 3 shows the frequency of sexual dysfunction detected by SFQ in each subgroup of sexual dysfunction. In our study $40.0 \%$ had sexual problems at least in one subgroup and $6.7 \%$ had problems in all subgroups.

To investigate various factors that may cause female sexual dysfunction, in a multivariate regression analysis, no significant relation was detected between female ages, male age, duration of marriage, male education, irregular menses, contraception methods used, BMI, hirsutism, and acne with FSD.

Table 1. Characteristics of female medical students who filled the Sexual Function Questionnaire

Mean \pm SD (minimum-maximum)

\begin{tabular}{ll}
\hline Female age $(\mathrm{y})$ & $24.30 \pm 1.29(21-29)$ \\
Husband's age $(\mathrm{y})$ & $27.03 \pm 2.94(22-34)$ \\
Duration of marriage (y) & $2.68 \pm 1.5(1-6)$ \\
BMI $\left(\mathrm{kg} / \mathrm{m}^{2}\right)$ & $22.76 \pm 2.99(17.85-30.04)$ \\
\hline
\end{tabular}

\begin{tabular}{ll}
\hline & Number $(\%)$ \\
\hline $\begin{array}{ll}\text { Husband education } \\
\text { Diploma }\end{array}$ & $2(6.7 \%)$ \\
University education & $28(93.3 \%)$ \\
& \\
Contraception method used & $5(16.7 \%)$ \\
Hormonal & $12(40 \%)$ \\
Non-hormonal & $13(43.3 \%)$ \\
Nothing & \\
Menses & $7(23.3 \%)$ \\
Irregular & $23(76.7 \%)$ \\
Regular &
\end{tabular}

\section{DISCUSSION}

The major factors that determine a female's sexuality are as follows: genetic and hormonal factors, learning of sexual components early in adulthood, suggestion of parents about the sexual function of children, religion, cultural factors, depression, physical disease and aging (4).

It is difficult to select a group that is unique for this type of study. We chose medical students as a match case group because age, health, and education are important factors in determining female sexual function. The group that we chose was in the third decade of life. The age shows its effects on sexual function in older subjects (11). So the bias of age effect is omitted in this way. Level of education affects the complaint of FSD in the same way. Well educated persons can understand items of the SFQ more readily and answer to questions without shame.

In 1999, the National Health and Social Life survey reported that $43 \%$ of American women reported sexual problems including: lack of desire, decreased vaginal lubrication, pain and discomfort with intercourse, or decreased pleasure and difficulty achieving orgasm (12). One study in 2004 revealed that $55 \%$ of the evaluated women were satisfied with their sexual life in the last month, $20 \%$ were fairly satisfied and $21 \%$ were unsatisfied (13). In a German study on female medical students, prevalence of sexual dysfunction was $25 \%$ (13). According to our research only $6.6 \%$ of women complained about sexual problems. The low prevalence of seeking medical care for sexual dysfunction is related to cultural issues in Iran.

After completing SFQ, we showed that $40.0 \%$ had sexual problems at least in one subgroup and $6.7 \%$ had problems in all subgroups. In comparison with nations with similar socio-cultural factors, Cayan et al reported a prevalence of $46.9 \%$ in Turkish women. Prevalence of FSD was $21.7 \%$ in the ages of $18-27$ years in the same study (11). Lower prevalence in young Turkish women and German medical students can be explained by difference in cultures. Shokrollahi et al studied the prevalence of sexual dysfunction in women seeking services at family planning centers in Tehran (8). There was at least one sexual dysfunction in $38 \%$ of women. Abnormality of desire, orgasm, lubrication and pain were found in $15 \%, 26 \%, 15 \%$ and $18 \%$ respectively. However in our study, these abnormalities were $20 \%$, $36.7 \%, 33.3 \%$ and $6.7 \%$ respectively. It seems that having more knowledge about the physiology of orgasm and lubrication in well-educated subjects can explain the higher prevalence of reporting abnormality in these two categories. Subjects with higher levels of education can explain some degrees of their pain as physiologic event, however any pain in general population may interpret and reported as pathologic.

In this pilot study we found no relation between demographic factors (female age, husband's age, husband's education, duration of marriage, hirsutism, acne, pattern of menses, contraception method utilized and BMI) and FSD, however it is postulated that many factors including age, knowledge, sexual knowledge, body image, partner dissatisfaction, and life stress (such as unemployment), chronic diseases, culture and ... have some effects on the prevalence of this disease. Shokrollahi et al reported that a positive correlation was found between sexual knowledge and the experience of orgasm $(r=0.1990, p=0.001)$. Cayan et al reported that sexual dysfunction was observed as significantly higher in the presence of older age $(p=0.001)$, lower educational level $(\mathrm{p}=0.012)$, chronic disease $(\mathrm{p}=0.032)$ 
Table 2. Guidelines to SFQ scores: SFQ score ranges indicative of likelihood of sexual dysfunction and normal function

\begin{tabular}{|c|c|c|c|}
\hline Domain & $\begin{array}{l}\text { High probability of } \\
\text { female sexual dysfunction }\end{array}$ & $\begin{array}{l}\text { Borderline probability of } \\
\text { normal sexual function }\end{array}$ & $\begin{array}{l}\text { High probability of } \\
\text { normal sexual function }\end{array}$ \\
\hline Desire & $5-16$ & $17-22$ & $23-31$ \\
\hline Arousal-sensation & $4-10$ & $11-13$ & $14-20$ \\
\hline Arousal-lubrication & $2-5$ & $6-7$ & $8-10$ \\
\hline Orgasm & $3-8$ & $9-11$ & $12-15$ \\
\hline Pain & $2-8$ & $9-11$ & $12-15$ \\
\hline Enjoyment & $6-16$ & $17-22$ & $23-30$ \\
\hline
\end{tabular}

Table 3. Prevalence of sexual dysfunction in relation to subgroups of sexual function

\begin{tabular}{lcccc}
\hline Domain & Mean \pm SD & $\begin{array}{c}\text { High probability of } \\
\text { female sexual dysfunction }\end{array}$ & $\begin{array}{c}\text { Borderline probability of } \\
\text { normal sexual function }\end{array}$ & $\begin{array}{c}\text { High probability of } \\
\text { normal sexual function }\end{array}$ \\
\hline Desire & $18.44 \pm 3.54$ & $6(20.0 \%)$ & $18(60.0 \%)$ & $3(10.0 \%)$ \\
\hline Arousal-sensation & $9.89 \pm 3.89$ & $17(56.7 \%)$ & $5(16.7 \%)$ & $7(23.3 \%)$ \\
\hline Arousal-lubrication & $6.20 \pm 2.32$ & $10(33.3 \%)$ & $9(30.3 \%)$ & $9(30.3 \%)$ \\
\hline Orgasm & $8.57 \pm 3.63$ & $11(36.7 \%)$ & $10(33.3 \%)$ & $7(23.3 \%)$ \\
\hline Pain & $9.96 \pm 2.22$ & $2(6.7 \%)$ & $26(86.7 \%)$ & 0 \\
\hline Enjoyment & $15.57 \pm 3.95$ & $6(20.0 \%)$ & $14(46.7 \%)$ & $8(26.7 \%)$ \\
\hline
\end{tabular}

$(8,11)$. It seems that the sample size in this study is too little to find these correlations. However the characteristics of this group are similar to the general population and by increasing the sample size valuable data will be obtained. For example in our research the incidence of hirsutism was $10 \%$ and acne $13.3 \%$. The incidence of acne is about $40 \%$ among girls under 17 and it decreases when they grow older. The incidence of hirsutism is $10 \%$ (14).

There is a wide variety of sexual problems with a high prevalence of different disorders in this selected population. It would be important to bring up the point that female medical students are involved in an emotionally-charged and physically-demanding program, which may affect their level of sexual dysfunction. Depending on how their living situation is, and whether they receive help in household chores, the pressure that hospital work and household work together entailed can have a toll on one's emotional state, and this is important to consider in light of the high prevalence of sexual dysfunction among female medical students. The continued quest to understand female sexual dysfunction requires more research on underlying medical and non-medical conditions.

\section{ACKNOWLEGEMENTS}

The authors would like to thank all medical students who participated.

\section{REFERENCES}

1. Morley JE, Kaiser FE. Female sexuality . Medical Clinics of North America 87(5):1077-1090; 2003

2. Imbimbo C, Gentile V, Palmieri A, et al. Female sexual dysfunction: an update on physiopathology. Journal of Endocrinology Investigation 26(3 suppl):102-4; 2003

3. Masters EH, Johnson VE. Human sexual response. Boston: little Brown; 1966

4. Fourcroy JL. Female sexual dysfunction: potential for pharmacotherapy. Drugs 63 (14):1445-1457; 2003

5. Marthol H, Hilz MJ. Female sexual dysfunction: a systemic overview of classification, pathophysiology, diagnosis and treatment. Fortschr Neurol Psychiatr 72 (3):121-35; 2004

6. Quirk FH, Heiman JR, Rosen RC, et al .Development of a Sexual Function Questionnaire for clinical trials of female sexual dysfunction. Journal of Women's Health and Gender Based Medicine 11(3):277-289; 2002

7. Simons J, Carey MP. Prevalence of sexual dysfunction: results from a decade of research .Arch Sex Behav 30(2):177-219. 2001

8. Shokrollahi P, Mirmohamadi M, Mehrabi F, et al. Prevalence of sexual dysfunction in women seeking services at family planning centers in Tehran. Journal of sex and marital therapy 25:211-215; 1999 
9. Rosen R, Brown C, Heiman J, et al. The female sexual function index (FSFI): a multidimentional self-report instrument for the assessment of female sexual function. Journal pf sex and marital therapy 26:191-208; 2000

10. Derogatis L, Rosen R, Leiblum S, et al. The female sexual distress scale (FSDS): initial validation of a standardized scale for assessment of sexually related personal distress in women. Journal of sex and marital therapy 28(4):317-330; 2002

11. Cayan S, Akbay E, Bozlu M, et al. The prevalence of female sexual dysfunction and potential risk factors that may impair sexual function in Turkish women. Urol Int 72(1):52-57; 2004
12. Laumann EO, Paik A, Rosen RC. Sexual dysfunction in the United States: prevalence and predictors. JAMA 281(6): 537-44; 1999

13. Dieckmann R, Oelke M, Uckert S, et al. The prevalence of sexual problems in female medical students. Urologe A 43(8): 955-962; 2004

14. Braunwald E, Fauci A.S, Kasper D.L, et al. Harisson's Principles of Internal Medicine.15th ed. New york(NY): Mc Grow Hill; 2001

Alaleh Asghari Roodsari is a 7th year medical student at Tehran University of Medical Sciences. Her research interests include Gynecology/Obstetrics and Infertility.

Afsaneh Khademi obtained her MD at Tabriz University, Iran and completed her Gynecology /Obstetrics at Tehran University of Medical Sciences. Her subspecialty training in infertility was at Infertility Department of Shariati Hospital.

Ehsan Akbari Hamed is a 7th year medical student at Tehran University of Medical Sciences. His research interests include Gynecology/Obstetrics, Infertility and drug abuse.

Seideh Leila Tabatabaeefar is a 7th year medical student at Tehran University of Medical Sciences. Her research interests includes Gynecology/obstetrics and Infertility. 